\title{
Walking through a virtual environment improves perceived size within and beyond the walked space
}

\author{
Zachary D. Siegel $^{1} \cdot$ Jonathan W. Kelly $^{1}$
}

Published online: 2 December 2016

(C) The Psychonomic Society, Inc. 2016

\begin{abstract}
Distances tend to be underperceived in virtual environments (VEs) by up to 50\%, whereas distances tend to be perceived accurately in the real world. Previous work has shown that allowing participants to interact with the VE while receiving continual visual feedback can reduce this underperception. Judgments of virtual object size have been used to measure whether this improvement is due to the rescaling of perceived space, but there is disagreement within the literature as to whether judgments of object size benefit from interaction with feedback. This study contributes to that discussion by employing a more natural measure of object size. We also examined whether any improvement in virtual distance perception was limited to the space used for interaction $(1-5 \mathrm{~m})$ or extended beyond $(7-11 \mathrm{~m})$. The results indicated that object size judgments do benefit from interaction with the VE, and that this benefit extends to distances beyond the explored space.
\end{abstract}

Keywords Spatial cognition · Visual perception

Virtual reality (VR) is an exciting technology, with applications including research, entertainment, training, and therapy. For VR to be fully effective, it must accurately represent the spatial properties of the environment. Previous work indicated that distances in virtual environments (VEs) are perceived to

Electronic supplementary material The online version of this article (doi:10.3758/s13414-016-1243-z) contains supplementary material, which is available to authorized users.

Zachary D. Siegel

zsiegel@iastate.edu

1 Department of Psychology, Iowa State University, W-112

Lagomarcino Hall, 901 Stange Road, Ames, IA 50011-1041, USA be about $70 \%$ of the intended distance (Waller \& Richardson, 2008). In contrast, real-world distances are often perceived accurately, at least when measured through action-based tasks (Loomis \& Knapp, 2003; but see Durgin \& Li, 2011).

Some researchers have attempted to improve distance perception in VR using a bottom-up approach by determining whether technological shortcomings prevent accurate perception. Researchers have examined the impacts of graphical quality (Thompson et al., 2004), field of view (Knapp \& Loomis, 2004), and display weight (Willemsen, Colton, Creem-Regehr, \& Thompson, 2009), but a clear bottom-up solution to underperception has yet to emerge.

In the present project, we examined a top-down approach designed to adapt the user to the VE, which has the potential to improve distance perception immediately rather than waiting until technology improves sufficiently. One common approach to improving distance perception in VR allows participants to interact with the VE while receiving visual feedback (Kelly, Donaldson, Sjolund \& Freiberg, 2013; Kelly, Hammel, Siegel, \& Sjolund, 2014; Mohler, Creem-Regehr \& Thompson, 2006; Richardson \& Waller, 2005, 2007; Waller \& Richardson, 2008). In a prototypical study by Waller and Richardson (2008), participants performed preinteraction distance judgments, followed by interaction and then postinteraction distance judgments. For pre- and postinteraction judgments, participants viewed a virtual object before walking to the perceived object location without feedback, referred to as a blind-walking distance judgment. Interaction involved walking through the VE with continuous visual feedback. Preinteraction judgments were around 50\% of the actual distance, whereas postinteraction judgments approached $100 \%$ of the actual distance. However, it is unclear, on the basis of these methods, whether the improvement was due to improved distance perception or visuomotor recalibration. 
Measurement of perceived size can be useful to discern whether walking interaction caused improvements in perceived distance. According to the size-distance invariance hypothesis (Sedgewick, 1986), perceived size and perceived distance are tightly coupled, and thus object size judgments can provide an alternative measure that is not susceptible to motor recalibration. However, Brenner and van Damme (1999) found that perceived object size, shape, and distance are largely independent. Although judgments of object size, shape, and distance were similarly affected by changes in perceived object distance, changes in perceived shape caused by motion parallax did not affect perceived size or distance, indicating their independence. Although the direct relationship between perceived size and distance has been questioned, judged distance and size have been shown to be highly correlated (Gogel, Loomis, Newman, \& Sharkey, 1985; Hutchison $\&$ Loomis, 2006), presumably due to the impact of perceived distance on both perceptual variables (Brenner \& van Damme).

Kelly et al. (2013) found that object size judgments increased after a walking interaction, consistent with an increase in perceived distance. More recently, Kunz, Creem-Regehr, and Thompson (2015) tested the effects of visual feedback, indicating faster- or slower-than-actual walking speed in a VE. Manipulation of visual speed impacted blind-walking but not size judgments. One explanation for the discrepant finding is that the participants in Kelly et al.'s study received feedback about the true walked distance, whereas Kunz et al.'s participants received false feedback about walking speed. Regardless, the equivocal results warrant further research to determine whether the effects of visual feedback during interaction cause rescaling of perceived space, visuomotor recalibration, or both.

For a top-down approach to be truly useful, the improvement in perceived distance should transfer beyond the distance walked during interaction. In an experiment by Kelly, Hammel, Siegel, and Sjolund (2014), participants made preand postinteraction blind-walking distance judgments to objects 1-5 m away. During walking interaction, participants walked with feedback to near (1 and $2 \mathrm{~m}$ ) or far (4 and $5 \mathrm{~m})$ objects. The postinteraction judgments in the near condition only improved for near distances, whereas the postinteraction judgments in the far interaction condition improved across near and far distances. It is possible, though, that walking interaction improved perceived distance for all distances up to but not beyond the farthest experienced during interaction. Alternatively, it is possible that walking interaction improved perceived distance for all distances, but that this broad improvement only occurred when participants received feedback on walked distances greater than $2 \mathrm{~m}$. Feedback received during walking interaction is likely based on an error signal representing the difference between the expected and the walked distance. The error signal on near interaction trials may not have been sufficiently large to cause rescaling across all distances. For example, a $20 \%$ underperception would cause a $1-\mathrm{m}$ distance to be perceived as $0.8 \mathrm{~m}$, whereas a 5 $\mathrm{m}$ distance would be perceived as $4 \mathrm{~m}$, leading to a larger error signal during interaction.

The two primary goals of the present project were to replicate past work showing that walking interaction causes improvements in perceived object size, and to determine whether such improvements generalize to distances beyond those experienced during interaction. For this study we utilized an intuitive size judgment task in which participants were first familiarized with a soccer ball in the real world. Once in VR, the participants were shown a randomly sized virtual soccer ball and tasked with correcting its size using adjustments with a controller. Size judgments were then used to calculate perceived distance under the assumption of size-distance invariance. As compared to blind walking, the resizing task served as a measure of perceived distance that should be immune to sensorimotor recalibration during walking interaction. As compared to verbal size judgments (Kelly et al., 2013), the resizing task should not be affected by an individual's skill in assigning metric values, and therefore judgments should be less variable. In light of the equivocal reports on the relationship between walking interaction and perceived size (Kelly et al., 2013; Kunz et al., 2015), the same experiment was conducted twice. Experiment $1 \mathrm{~b}$ directly replicated Experiment 1a. For ease of exposition, the two experiments are described together.

\section{Method}

\section{Participants}

Groups of 33 (Exp. 1a) and 53 (Exp. 1b) undergraduate students from Iowa State University participated for course credit. Six (Exp. 1a) and four (Exp. 1b) participants were removed from all analyses due to equipment failure. One additional participant (Exp. 1a) was removed from all analyses for reporting artificially shortened walking for fear of hitting physical objects.

\section{Stimuli}

The VE was displayed on an nVisor SX111 monitor (NVIS, Reston, VA) with a $102^{\circ} \times 64^{\circ}$ field of view. Vizard software (WorldViz, Santa Barbara, CA) was used to render the graphics. The VE consisted of an endless plane with a grass texture and gray sky (Fig. 1). 

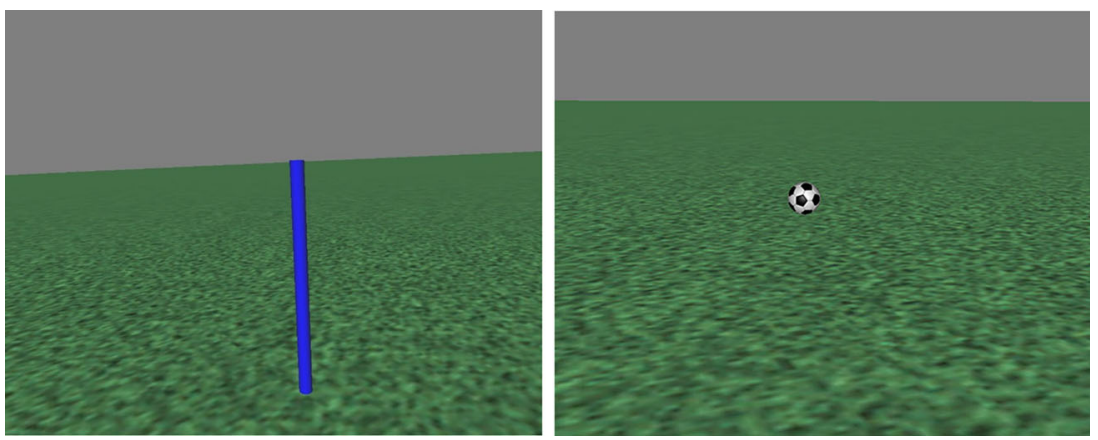

Fig. 1 Virtual environment with the stimuli used in the blind-walking task (left) and the resizing task (right)

\section{Design}

We collected two different measures of perceived distance: object resizing and blind walking. Both measures were performed both before and after walking interaction (object resizing was performed before blind walking). Experiment $1 \mathrm{~b}$ also included a verbal measure, but the results were inconclusive and are not discussed further.

In the resizing task, participants manipulated the size of a virtual soccer ball (Fig. 1) until it appeared correct. On each trial, the initial soccer ball size was randomly selected between $30 \%$ and $300 \%$. Resizing was performed three times at five distances, resulting in 15 trials. Distances 1,3 , and $5 \mathrm{~m}$ (near) overlapped with the distances used in walking interaction. Distances 7 and $11 \mathrm{~m}$ (far) extended beyond the distances used in walking interaction.

In the blind-walking task, participants judged the distance to a vertical post (Fig. 1; 10-cm diameter and scaled to the participant's eye height) at each of five distances (1, 2, 3, 4, and $5 \mathrm{~m}$ ). Three repetitions of each distance resulted in 15 trials.

Participants interacted with the VE by walking with continuous visual feedback to a vertical blue post (the same post used in the blind-walking task). Interaction was performed three times at five distances $(1,2,3,4$, and $5 \mathrm{~m})$, resulting in 15 trials.

For all experimental tasks (resizing, blind walking, and interaction), only one object at a time was visible within the VE, preventing comparison across trials. Furthermore, trials within a task were presented in a random sequence.

In summary, the dependent variables were size and blindwalking judgments. The independent variables were egocentric object distance and whether the perceptual judgments occurred before (pre) or after (post) interaction. Both independent variables were manipulated within participants.

\section{Procedure}

Participants were given verbal instructions on the blindwalking and resizing tasks. They were also shown a real soccer ball and allowed to hold it for the duration of the instructions. Participants then donned the head-mounted display (HMD) and were led to the viewing location.

In the resizing task, participants viewed a soccer ball and used joystick buttons to increase or decrease the ball size until it appeared correct. Resizing was always performed while standing at the viewing location (participants never walked to the virtual ball).

In the blind-walking task, participants viewed a blue target post for $5 \mathrm{~s}$, after which the scene vanished and they attempted to walk to the perceived location. After each blind-walking trial, participants were guided by the experimenter back to the viewing location.

Participants interacted with the VE by walking, with vision, to the blue post. Upon reaching the target, the scene vanished and participants were guided back to the viewing location.

\section{Analysis}

Distance judgments were analyzed as a ratio of judged/actual distance, consistent with much past work (Kelly et al., 2013; Kelly et al., 2014; Kunz et al., 2015; Mohler et al., 2006; Richardson \& Waller, 2005, 2007; Waller \& Richardson, 2008). Furthermore, the effect of walking interaction on judged distance can be described as a constant ratio change from pre- to posttest (Kelly et al., 2014).

Size judgments showed evidence of anchoring, whereby responses were biased toward the initial object size on a given trial. To correct for anchoring, the judged size was first expressed as the ratio of the judged to the correct size. Next, the mean ratio was subtracted from all size judgment ratios, which were then regressed against the initial size (see Supplemental Fig. S1). The resulting linear equations generally fit the data well $\left(R^{2}\right.$ ranging from .72 to .89$)$ and were used to adjust the size judgment ratios in order to correct for the anchoring bias. Correction was done by applying the linear equation (relating size judgment bias to initial object size) to size judgments based on the initial ball size for that trial. Correction was done separately for each experiment, and for pre- and postinteraction judgments. 
After correcting for anchoring, the size judgments were converted to ratios of judged to actual distance, based on the size-distance invariance hypothesis (Sedgewick, 1986). According to this hypothesis, perceived object distance $\left(D^{\prime}\right)$ is directly related to the perceived object size $\left(S^{\prime}\right)$ and object angular size $(\alpha)$ :

$D^{\prime}=S^{\prime} / \tan (\alpha)$.

The perceived size was always $22 \mathrm{~cm}$ (the participant's task was to adjust the virtual ball until it appeared to be the same size as a real soccer ball, which is $22 \mathrm{~cm}$ in diameter). Angular size $(\alpha)$ was determined by the adjusted size of the soccer ball $(S)$, along with the actual distance of the soccer ball $(D)$ :

$\alpha=\operatorname{atan}(S / D)$.

The perceived distance was then divided by the actual distance to produce a ratio of judged-to-actual distance.

\section{Results}

The distance judgment ratios for blind-walking and size judgments in Experiments $1 \mathrm{a}$ and $1 \mathrm{~b}$ are shown in Fig. 2 (Supplemental Fig. S2 shows the distance and size judgments as a function of actual object distance). Planned comparisons were conducted to evaluate whether walking interaction improved blind-walking distance judgments and whether walking interaction improved size judgments in near and in far space.

In Experiment 1a, the blind-walking judgments improved from pretest $(M=.63, S D=.10)$ to posttest $(M=.76, S D=$ $.17), t(25)=4.77, p<.001, \eta_{\mathrm{p}}{ }^{2}=.48$, and this effect was

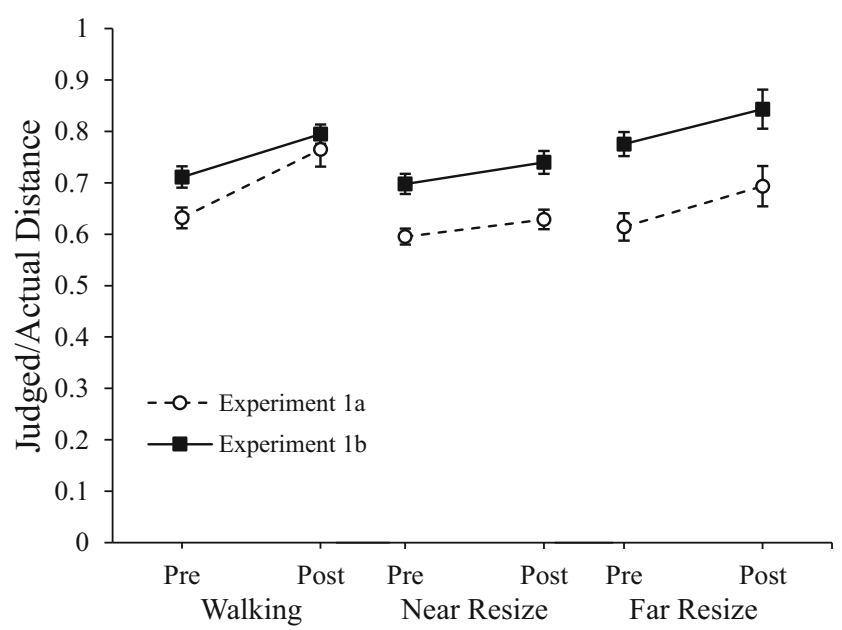

Fig. 2 Ratios of judged to actual distance in Experiments 1a and 1b. Error bars represent \pm 1 standard error based on the between-participants variance replicated in Experiment 1b, in which blind-walking judgments also improved from pretest $(M=.71, S D=.15)$ to posttest $(M=.80, S D=.13), t(48)=5.70, p<.001, \eta_{\mathrm{p}}{ }^{2}=$ .40. In Experiment 1a, near $(1,3$, and $5 \mathrm{~m})$ resizing judgments improved from pretest $(M=.57, S D=.08)$ to posttest $(M=$ $.63, S D=.10), t(25)=2.55, p=.017, \eta_{\mathrm{p}}{ }^{2}=.21$, and this effect was replicated in Experiment $1 \mathrm{~b}$, in which near resizing judgments improved from pretest $(M=.70, S D=.12)$ to posttest $(M=.73, S D=.12), t(48)=3.44, p=.001, \eta_{\mathrm{p}}{ }^{2}=.20 . \mathrm{In}$ Experiment 1a, far (7 and $11 \mathrm{~m})$ resizing judgments improved from pretest $(M=.61, S D=.14)$ to posttest, $(M=.69, S D=$ $.20), t(25)=2.68, p=.013, \eta_{\mathrm{p}}{ }^{2}=.22$, and this effect was replicated in Experiment 1b, in which far resizing judgments likewise improved from pretest $(M=.77, S D=.21)$ to posttest $(M=.84, S D=.27), t(48)=2.01, p=.050, \eta_{\mathrm{p}}{ }^{2}=.19$.

\section{Discussion}

Walking through the VE with continuous visual feedback caused improvement in blind-walking distance judgments and object size judgments. Furthermore, size judgments improved for object distances experienced during walking interaction and for distances beyond the interaction space. These findings were consistent across both experiments and serve to replicate past research, as well as to extend the understanding of space perception in VEs.

These experiments replicated past work showing that walking interaction causes improvement in judgments of distance (Waller \& Richardson, 2008) and size (Kelly et al., 2013). The finding that size judgments increased after interaction indicates that walking interaction leads to a rescaling of perceived space rather than to simple recalibration of walked distance. However, the size judgment results also appear contradictory to results reported by Kunz et al. (2015). Possible explanations are discussed later in this section.

In addition to the aforementioned replications, these experiments add new data showing that walking interaction causes improvements in judged size for objects located beyond the walking space. This is somewhat surprising in light of the research reported by Kelly et al. (2014), in which blindwalking judgments did not improve for distances beyond those experienced during walking interaction. However, in that study, the participants only received feedback while walking to distances of 1-2 $\mathrm{m}$, as compared to $1-5 \mathrm{~m}$ in the present study. It is therefore possible that longer distances are necessary to produce improvement beyond the interaction space. It is also possible that such broad improvement is specific to size perception and would not be found in blind-walking judgments, but this possibility was not tested in the present project, due to physical space limitations.

The finding that size judgments improved after walking interaction appears contradictory to results reported by Kunz 
et al. (2015). In their experiment, participants performed blind-walking and object size judgments before and after an interaction in which visual walking speed was manipulated. Slower-than-actual visual movement caused longer blindwalking judgments, and faster-than-actual visual movement caused shorter blind-walking judgments. These same manipulations had no effect on size judgments, suggesting that the visual feedback provided during walking does not cause perceptual rescaling. Although there are several methodological differences across these studies, we believe the critical difference is that the participants in the present experiments and in Kelly et al. (2013) received feedback about walked distance rather than walking speed. The method used by Kunz et al. is conceptually similar to experiments reported by Rieser, Pick, Ashmead, and Garing (1995), in which participants walked on a treadmill pulled behind a tractor, allowing independent manipulation of body-based and visual cues to walking speed. In those experiments, the effect of manipulating visual speed was specific to walking judgments, and did not generalize to other measures of perceived space, such as ball throwing. It is therefore possible that the discrepancy between the findings reported here and those of Kunz et al. is due to differences in the interactions: Manipulation of visual walking speed may only recalibrate walked distance, whereas feedback regarding walked distance causes a rescaling of perceived space.

The resizing task was chosen over the verbal size judgments used in past work (e.g., Kelly et al., 2013) because resizing should not be affected by individual skill in assigning metric values, making judgments less variable. To evaluate this possibility, within-participants standard deviations of size judgments were calculated for each participant at each test distance. The standard deviations were then averaged across these distances. The within-participants standard deviations in the resizing task $(M=.072, S D=.050)$ were smaller than those in the verbal task $(M=.14, S D=.07)$ used by Kelly et al. (2013) $[t(97)=3.14, p=.002]$. Between-participants standard deviations in the overall size judgments were calculated by first determining the between-participants standard deviation of the distance judgment ratios for each test distance, and then averaging those standard deviations. The between-participants standard deviation in the resizing task (.16) was $47 \%$ smaller than the one in the verbal task (.30).

Kelly et al. (2013) proposed that walking interaction leads to perceptual learning, whereby the visual system assigns higher weights to distance cues that are more predictive of actual distance in the VE. For example, texture gradient can be reliably reproduced in the VE, whereas collimating lenses in the HMD fix the accommodative state of the lens at a specific distance, rendering it useless as a distance cue. According to this theory, the effect of walking interaction on perceived distance will only transfer to another VE or HMD if the predictive values of the distance cues remain the same across contexts.
Experiment $1 \mathrm{~b}$ was a direct replication of Experiment $1 \mathrm{a}$, in that the tasks and lab equipment were identical, as was the population from which the participant samples were recruited. Despite those similarities, walking $[F(1,73)=3.17, p=.08$, $\left.\eta_{\mathrm{p}}{ }^{2}=.04\right]$ and size $\left[\right.$ near: $F(1,73)=12.78, p=.001, \eta_{\mathrm{p}}{ }^{2}=.15$; far: $\left.F(1,73)=11.19, p=.001, \eta_{\mathrm{p}}{ }^{2}=.13\right]$ judgments were overall larger in Experiment $1 \mathrm{~b}$ than in Experiment 1a. Although some aspects varied across the experiments (e.g., time of year, the researchers conducting the study), none provide insight into the overall difference across experiments.

The experiments reported here weigh in on disparate findings in the literature regarding perceptual changes caused by interaction with a VE. These results indicate that continuous visual feedback regarding walked distance causes a rescaling of perceived space. Additionally, rescaling can extend beyond the range of space experienced during interaction. Underperception of distance in VR continues to be a problem for users and researchers alike, but improvements through interacting with the VE show potential as a solution until technology advances sufficiently.

\section{References}

Brenner, E., \& van Damme, W. J. M. (1999). Perceived distance, shape and size. Vision Research, 39, 975-986.

Durgin, F. H., \& Li, Z. (2011). Perceptual scale expansion: An efficient angular coding strategy for locomotor space. Attention, Perception, \& Psychophysics, 73, 1856-1870. doi:10.3758/s13414-011-0143-5

Gogel, W. C., Loomis, J. M., Newman, N. J., \& Sharkey, T. J. (1985). Agreement between indirect measures of perceived distance. Perception \& Psychophysics, 37, 17-27.

Hutchison, J. J., \& Loomis, J. M. (2006). Does energy expenditure affect the perception of egocentric distance? A failure to replicate Experiment 1 of Proffitt, Stefanucci, Banton, and Epstein (2003). Spanish Journal of Psychology, 9, 332-339.

Kelly, J. W., Donaldson, L. S., Sjolund, L. A., \& Freiberg, J. B. (2013). More than just perception-action recalibration: Walking through a virtual environment causes rescaling of perceived space. Attention, Perception, \& Psychophysics, 75, 1473-1485. doi:10.3758/s13414013-0503-4

Kelly, J. W., Hammel, W., Siegel, Z. D., \& Sjolund, L. A. (2014). Recalibration of perceived distance in virtual reality occurs rapidly and transfers asymmetrically across scale. IEEE Transactions on Visualization and Computer Graphics, 20, 588-595.

Knapp, J. M., \& Loomis, J. M. (2004). Limited field of view of headmounted displays is not the cause of distance underestimation in virtual environments. Presence: Teleoperators and Virtual Environments, 13, 572-577.

Kunz, B. R., Creem-Regehr, S. H., \& Thompson, W. B. (2015). Testing the mechanisms underlying improved distance judgments in virtual environments. Perception, 44, 446-453. doi:10.1068/p7929

Loomis, J. M., \& Knapp, J. M. (2003). Visual perception of egocentric distance in real and virtual environments. In L. J. Hettinger \& M. W. Haas (Eds.), Virtual and adaptive environments (pp. 21-46). Mahwah: Erlbaum.

Mohler, B. J., Creem-Regehr, S. H., \& Thompson, W. B. (2006). The influence of feedback on egocentric distance judgments in real and 
virtual environments. In Proceedings of the 3rd Symposium on Applied Perception in Graphics and Visualization (pp. 9-14). New York: ACM Press.

Richardson, A. R., \& Waller, D. (2005). The effect of feedback training on distance estimation in virtual environments. Applied Cognitive Psychology, 19, 1089-1108.

Richardson, A. R., \& Waller, D. (2007). Interaction with an immersive virtual environment corrects users' distance estimates. Human Factors, 49, 507-517.

Rieser, J. J., Pick, H. L., Ashmead, D. H., \& Garing, A. E. (1995). Calibration of human locomotion and models of perceptual-motor organization. Journal of Experimental Psychology: Human Perception and Performance, 21, 480-497. doi:10.1037/00961523.21.3.480

Sedgewick, H. A. (1986). Space perception. In K. R. Boff, L. Kaufman, \& J. P. Thomas (Eds.), Handbook of perception and human performance (Sensory processes and perception, Vol. 1, pp. 21.121.57). New York: Wiley.

Thompson, W. B., Willemsen, P., Gooch, A., Creem-Regehr, S. H., Loomis, J. M., \& Beall, A. C. (2004). Does the quality of the computer graphics matter when judging distances in visually immersive environments? Presence: Teleoperators and Virtual Environments, $13,560-571$.

Waller, D., \& Richardson, A. R. (2008). Correcting distance estimates by interacting with immersive virtual environments: Effects of task and available sensory information. Journal of Experimental Psychology: Applied, 14, 61-72. doi:10.1037/1076-898X.14.1.61

Willemsen, P., Colton, M. B., Creem-Regehr, S. H., \& Thompson, W. B. (2009). The effects of head-mounted display mechanical properties and field of view on distance judgments in virtual environments. ACM Transactions on Applied Perception, 6(8), 1-14. doi:10.1145 /1498700.1498702 